\title{
THE LIFT INVARIANT DISTINGUISHES COMPONENTS OF HURWITZ SPACES FOR $A_{5}$
}

\author{
ADAM JAMES, KAY MAGAARD, AND SERGEY SHPECTOROV \\ (Communicated by Pham Huu Tiep)
}

\begin{abstract}
Hurwitz spaces are moduli spaces of curve covers. The isomorphism classes of covers of $P^{1} \mathbb{C}$ with given ramification data are parameterized combinatorially by Nielsen tuples in the monodromy group $G$. The Artin braid group acts on Nielsen tuples, and the orbits of this action correspond to the connected components of the corresponding Hurwitz space. In this article we consider the case $G=A_{5}$. We give a complete classification of the braid orbits for all ramification types, showing that the components are always distinguishable by the Fried-Serre lift invariant.
\end{abstract}

\section{INTRODUCTION}

Let $X$ be a compact Riemann surface of genus $g$. Let $\phi: X \rightarrow P^{1} \mathbb{C}$ be a degree $n$ meromorphic function. For all but finitely many points $x \in P^{1} \mathbb{C}$, the fibre $\phi^{-1}(x)$ has cardinality $n$. We call those points, whose fibre has cardinality strictly less than $n$, branch points of $\phi$, and we let $B=B_{\phi}=\left\{b_{1}, \ldots, b_{r}\right\}$ be the set of branch points of $\phi$.

For any $x \in P^{1} \mathbb{C} \backslash B$, the fundamental group $\pi_{1}\left(P^{1} \mathbb{C} \backslash B, x\right)$ acts transitively on the $n$ elements of the fibre. This action is known as the monodromy action, and it induces a homomorphism

$$
\phi_{*}: \pi_{1}\left(P^{1} \mathbb{C} \backslash B, x\right) \rightarrow S_{n} .
$$

The image of $\phi_{*}$ is called the monodromy group of $\phi$. The function $\phi$ and the basepoint $x$ determine the monodromy group. Changing the basepoint results in a conjugate monodromy group. For every $1 \leq i \leq r$, let $\gamma_{i}$ be the closed curve winding once around the point $b_{i}$. Then $\pi_{1}\left(P^{1} \mathbb{C} \backslash B, x\right)$ is generated by the homotopy classes of the $\gamma_{i}$ (which we also denote by $\gamma_{i}$ ), and the $\gamma_{i}$ satisfy the single relation

$$
\gamma_{1} \cdots \gamma_{r}=1
$$

The function $\phi_{*}$ takes the generators $\gamma_{1}, \ldots, \gamma_{r}$ to non-identity elements $g_{1}, \ldots, g_{r}$, which generate $G$ and which themselves satisfy the product-one condition:

$$
g_{1} \cdots g_{r}=1 .
$$

If $C_{i}$ denotes the conjugacy class of $g_{i}$ then the tuple $\boldsymbol{C}=\left(C_{1}, \ldots, C_{r}\right)$ is called the ramification type (or simply type) of $f$.

Received by the editors October 12, 2012 and, in revised form, February 25, 2013.

2010 Mathematics Subject Classification. Primary 20B25, 20B40; Secondary 14H55, 20F36, $14 \mathrm{H} 10$.

(C) 2014 American Mathematical Society Reverts to public domain 28 years from publication 
Consider the set of all possible monodromy homomorphisms of the form (11). Each homomorphism is determined by the images of the standard generators. Hence the set of monodromy homomorphisms for a fixed group $G$ is given by

$$
N(G)=\left\{\left(g_{1}, \ldots, g_{r}\right) \mid g_{i} \neq 1,1 \leq i \leq r,\left\langle g_{1}, \ldots, g_{r}\right\rangle=G \text { and } g_{1} \cdots g_{r}=1\right\} .
$$

A tuple $\left(g_{1}, \ldots, g_{r}\right) \in N(G)$ is called a Nielsen tuple (or Hurwitz tuple). If $\boldsymbol{g}=$ $\left(g_{1}, \ldots, g_{r}\right)$ and $\boldsymbol{g}^{\prime}=\left(g_{1}^{\prime}, \ldots, g_{r}^{\prime}\right)$ are two Nielsen tuples such that there exists $h \in G$ satisfying $g_{i}^{h}=g_{i}^{\prime}$ for all $i$, then we write $\boldsymbol{g}^{h}=\boldsymbol{g}^{\prime}$. Conjugate tuples correspond to equivalent monodromy homomorphisms. Often we consider tuples up to conjugation since our choice of basepoint is arbitrary.

Let $\mathcal{B}_{r}=\left\langle Q_{1}, \ldots, Q_{r-1}\right\rangle$ be the Artin braid group on $r$ strands (see [1]), with the generator $Q_{i}$ crossing strand $i$ under strand $i+1$. Consider the configuration space

$$
\mathcal{C}(\mathbb{C}, r)=\mathcal{F}(\mathbb{C}, r) / S_{r},
$$

where

$$
\mathcal{F}(\mathbb{C}, r)=\left\{\left(c_{1}, \ldots, c_{r}\right) \in \mathbb{C}^{r} \mid c_{i} \neq c_{j} \text { for } i \neq j\right\}
$$

and $S_{r}$ is the symmetric group acting by permuting the entries in the tuple. Points of $\mathcal{C}(\mathbb{C}, r)$ can be viewed as subsets of $\mathbb{C}$ of cardinality $r$. Pick an arbitrary basepoint $\boldsymbol{c}=\left\{c_{1}, \ldots, c_{r}\right\} \in \mathcal{C}(\mathbb{C}, r)$. The fundamental group $\pi_{1}(\mathcal{C}(\mathbb{C}, r), \boldsymbol{c})$ is isomorphic to $\mathcal{B}_{r}$.

Each strand of a braid $Q \in \mathcal{B}_{r}$ connects some initial point $c_{i} \in \boldsymbol{c}$ to some terminal point $c_{j} \in \boldsymbol{c}$. This defines a permutation $\sigma_{Q}$ of $\{1, \ldots, r\}$ (and of $\boldsymbol{c}=\left\{c_{1}, \ldots, c_{r}\right\}$ ). The mapping $Q \mapsto \sigma_{Q}$ is a homomorphism. The kernel of this homomorphism, called the pure braid group and denoted $\mathcal{P} \mathcal{B}_{r}$, consists of all those braids which do not permute $\boldsymbol{c}$. Another way to look at $\mathcal{P} \mathcal{B}_{r}$ is that it is naturally isomorphic to $\pi_{1}(\mathcal{F}(\mathbb{C}, r), \boldsymbol{f})$, where $\boldsymbol{f}$ is the (ordered) tuple $\left(c_{1}, \ldots, c_{r}\right)$, much in the same way as $\mathcal{B}_{r}$ is isomorphic to $\pi_{1}(\mathcal{C}(\mathbb{C}, r), c)$. The pure braid group is generated by

$$
P_{i, j}=Q_{i} \cdots Q_{j-2} Q_{j-1}^{2} Q_{j-2} \cdots Q_{i}, \quad 0<i<j \leq r .
$$

Given a tuple $\boldsymbol{g}=\left(g_{1}, \ldots, g_{r}\right) \in N(G)$, the generator $Q_{i} \in \mathcal{B}_{r}$ acts on $\boldsymbol{g}$ as follows:

$$
Q_{i}(\boldsymbol{g})=\left(g_{1}, \ldots, g_{i-1}, g_{i} g_{i+1} g_{i}^{-1}, g_{i}, \ldots, g_{r}\right),
$$

and the pure generator $P_{i, j} \in \mathcal{P} \mathcal{B}_{r}$ acts on $\boldsymbol{g}$ by

$$
P_{i, j}(\boldsymbol{g})=\left(g_{1}, \ldots, g_{i-1}, g_{i}^{\left(g_{i} \cdots g_{j}\right)^{-1}}, g_{i+1}^{g_{i}^{-1}}, \ldots, g_{j}^{g_{i}^{-1}}, g_{j+1}, \ldots, g_{r}\right) .
$$

Two Nielsen tuples, $\boldsymbol{g}, \boldsymbol{g}^{\prime}$, are said to be braid equivalent if there exists $Q \in \mathcal{B}_{r}$ such that $Q(\boldsymbol{g})=\boldsymbol{g}^{\prime}$. For non-identity conjugacy classes $C_{1}, \ldots, C_{r}$ of $G$, let

$$
N(G, \boldsymbol{C})=\left\{\boldsymbol{g} \in N(G) \mid g_{i} \in C_{\sigma(i)} \text { for some } \sigma \in S_{r} \text { and all } i\right\} .
$$

Define the (inner) Nielsen class corresponding to the ramification type $\boldsymbol{C}=$ $\left(C_{1}, \ldots, C_{r}\right)$ to be

$$
\mathrm{Ni}^{\text {in }}(G, C)=N(G, C) / \operatorname{Inn}(G) .
$$

The braid group $\mathcal{B}_{r}$ acts on $N(G)$ and hence on $\mathrm{Ni}^{\mathrm{in}}(G, \boldsymbol{C})$. We call orbits under the braid group action braid orbits. This braiding action commutes with conjugation by an element of $G$ and so we have an action of $\operatorname{Inn}(G) \times \mathcal{B}_{r}$ on Nielsen tuples. Note that the class $\mathrm{Ni}^{\text {in }}(G, C)$ does not depend on the order in which classes appear in $\boldsymbol{C}$. Hence, in this context, types can be treated as multisets. In particular, 
we can take $\boldsymbol{C}$ to be in ascending order with respect to some standardized ordering of conjugacy classes (such as in [5]).

For a particular group $G$ and type $C$, the braid orbits (and in higher genus the mapping class orbits) are known, amongst other things (see 12, for example), to classify components of the Hurwitz space. The inner Hurwitz space, denoted $\mathcal{H}^{\text {in }}(G, C)$, is a variety parameterizing meromorphic functions on genus $g$ Riemann surfaces with ramification type $C$ and monodromy group $G$ (see [8, 16, 17]). Note that the genus $g$ is not an independent parameter and can be computed for a given group $G$ and type $\boldsymbol{C}$ from the Riemann-Hurwitz formula.

Remark 1.1. Care must be taken when discussing Hurwitz spaces and their different variants. Depending on the geometric properties of interest one may choose to consider different notions of equivalence for monodromy homomorphisms.

The equivalence used in the present article is inner equivalence, this coming from our decision to consider tuples up to the action of an inner automorphism. Alternatively, one may wish to consider tuples up to absolute equivalence. Let $\operatorname{Abs}(G, C)$ denote the subgroup of automorphisms of $G$ preserving the type, i.e.,

$$
\operatorname{Abs}(G, C)=\left\{\iota \in \operatorname{Aut}(G) \mid \iota\left(C_{i}\right)=C_{\pi(i)} \text { for some } \pi \in S_{r} \text { and all } 1 \leq i \leq r\right\} .
$$

The absolute Nielsen class of $G$ is

$$
\mathrm{Ni}^{\mathrm{abs}}(G, \boldsymbol{C})=N(G, \boldsymbol{C}) / \operatorname{Abs}(G) .
$$

The orbits of the braid group on $\mathrm{Ni}^{\text {abs }}(G, C)$ correspond to components of the absolute Hurwitz space $\mathcal{H}^{\text {abs }}(G, C)$. Both the inner and absolute Hurwitz spaces can be defined as varieties over $\mathbb{Q}$ and there exists an algebraic morphism

$$
\mathcal{H}^{\text {in }}(G, C) \rightarrow \mathcal{H}^{\text {abs }}(G, C)
$$

defined over $\mathbb{Q}([8])$.

In general, little is known about the components of the Hurwitz spaces for a given group $G$. The earliest result concerning the irreducibility of Hurwitz spaces, due to Clebsch, says that if $G=S_{n}$ and $\boldsymbol{C}=\left(C_{1}, \ldots, C_{r}\right)$, where every $C_{i}$ is the class of transpositions, then $\mathrm{Ni}^{\text {in }}(G, C)$ is non-empty if and only if $r$ is even and $r \geq 2(n-1)$. Furthermore, if this holds, then there is exactly one braid orbit, and hence $\mathcal{H}^{\text {in }}\left(S_{n}, C\right)$ is irreducible ([4]). Fried has shown that if $G=A_{n}$ and the type consists solely of 3-cycles, then, apart from some small cases, both the absolute and inner Hurwitz spaces have two components. Moreover, Fried introduced an invariant, called the Fried-Serre lift invariant, showing that this invariant separates the braid orbits. The key idea of the lift invariant is that we may lift a tuple in a group $G$ to a tuple in a covering group $\widehat{G}$ of $G$.

For Fried's particular example, where $G$ is an alternating group, the covering groups are well understood. Whilst investigating projective representations of the alternating groups, Schur showed that $A_{n}$ has a unique double cover $2 \cdot A_{n}$. Moreover, every odd order element $g$ in $A_{n}$ has a unique odd order lift, which we shall denote by $\hat{g}$.

Definition 1.2. Let $\boldsymbol{g}=\left(g_{1}, \ldots, g_{r}\right)$ be a Nielsen tuple in $A_{n}$ consisting of odd order elements. Let $\hat{\boldsymbol{g}}=\left(\hat{g_{1}}, \ldots, \hat{g_{r}}\right)$ be the lifted tuple in $2 \cdot A_{n}$. The Fried-Serre lift invariant of $t$, written $\operatorname{LI}(t)$, is

$$
\mathrm{LI}(t)=\hat{g_{1}} \cdots \hat{g_{r}} .
$$


The lift invariant is a preimage of 1 and is hence central in $\widehat{G}$. For convenience we identify the centre with the multiplicative group $\{1,-1\}$. The lift invariant takes values in this set. The lift invariant is an invariant of the braid orbit [7, and is not defined for types involving classes of elements of even order.

Liu and Osserman have studied absolute Hurwitz spaces with $g=0, G$ a transitive subgroup of $S_{n}$, and type consisting of conjugacy classes which are pure-cycle, showing that under these circumstances there is exactly one component ([13]). Fried's small exceptional cases are genus 0 and therefore complement the result of Liu and Osserman. Two different classifications describe the inner Hurwitz spaces for dihedral groups (see [3], 2] and [15]).

Throughout this article we use the following shorthand for the conjugacy classes of $A_{5}$ :

- $2 A=(1,2)(3,4)^{A_{5}}$,

- $3 A=(1,2,3)^{A_{5}}$,

- $5 A=(1,2,3,4,5)^{A_{5}}$,

- $5 B=(1,2,3,5,4)^{A_{5}}$.

The group $A_{5}$ has a non-trivial outer automorphism which permutes the two classes of 5-cycles but fixes all other conjugacy classes. This automorphism corresponds to conjugation by $(4,5) \in S_{5}$. For each conjugacy class $C$ of $G$, let $n_{C}=n_{C}(\boldsymbol{C})$ denote the number of repetitions of the conjugacy class $C$ within the type $\boldsymbol{C}$. Let $S(\boldsymbol{C})$ be the vector of frequencies of the conjugacy classes amongst the type:

$$
S(\boldsymbol{C})=\left(n_{2 A}, n_{3 A}, n_{5 A}, n_{5 B}\right) .
$$

The vector of frequencies identifies $C$ up to a permutation.

The main result of this article is the following.

Main Theorem. For $G=A_{5}$ and a type $\boldsymbol{C}=\left(C_{1}, \ldots, C_{r}\right), r \geq 3$, the Nielsen class $\mathrm{Ni}^{\mathrm{in}}(G, C)$ is non-empty if and only if $\boldsymbol{C}$ is on List $A$. Given that, there are at most two braid orbits on $\mathrm{Ni}^{\text {in }}(G, C)$; furthermore,

- if $n_{2 A}>0$, then $\mathcal{B}_{r}$ acts transitively on $\mathrm{Ni}^{\mathrm{in}}(G, C)$;

- if $n_{2 A}=0$, then there are two braid orbits of $\mathrm{Ni}^{\mathrm{in}}(G, \boldsymbol{C})$ if and only if $\boldsymbol{C}$ is not on List B; moreover, the two orbits are distinguished by the Fried-Serre lift invariant.

Note that the lists shown are in condensed form. The actual Lists A and B are obtained from the condensed form by permutations and the outer automorphism.

The Main Theorem offers the first complete description of all braid orbits for a non-soluble group. This breakthrough follows considerable computational effort culminating in the calculation of the list of exceptional types. These computational methods, introduced in [14] and extended in [11, allow us to complete our proof via an inductive argument.

The following is a translation of the Main Theorem into the language of Hurwitz spaces. Whilst the statement is given in terms of inner Hurwitz spaces, it should be noted that the lift invariant is preserved under the action of the absolute group. Therefore, the result below may be translated into a result concerning absolute Hurwitz spaces.

Corollary. For $G=A_{5}$ and a type $\boldsymbol{C}$ of length at least 3, the Hurwitz space $\mathcal{H}^{\text {in }}\left(A_{5}, \boldsymbol{C}\right)$ is non-empty if and only if $\boldsymbol{C}$ is not in List $A$. Furthermore, if nonempty, $\mathcal{H}^{\text {in }}\left(A_{5}, \boldsymbol{C}\right)$ is connected if and only if $\boldsymbol{C}$ is in List $B$ or $n_{2 A}>0$. In all 
TABle 1. List $A$ : Types $\boldsymbol{C}$, up to permutation and outer automorphism, for which the Nielsen class $\mathrm{Ni}^{\text {in }}\left(A_{5}, \boldsymbol{C}\right)$ is empty. For each type we also list the value of genus coming from the RiemannHurwitz formula.

List B: Odd types, also up to permutation and outer automorphism, for which only one braid orbit on $\mathrm{Ni}^{\text {in }}\left(A_{5}, C\right)$ exists; the middle column shows the lift invariant of the orbit. The right column lists the genus of the covering space.

(a)

\begin{tabular}{lr} 
Type & Genus \\
\hline$(2 A, 2 A, 2 A)$ & -1 \\
$(2 A, 2 A, 3 A)$ & -1 \\
$(2 A, 2 A, 5 A)$ & 0 \\
$(2 A, 3 A, 3 A)$ & -1 \\
$(2 A, 5 A, 5 A)$ & 1 \\
$(3 A, 3 A, 3 A)$ & -1 \\
$(5 A, 5 A, 5 B)$ & 2 \\
$(2 A, 2 A, 2 A, 2 A)$ & 0
\end{tabular}

(b)

\begin{tabular}{lrc} 
Type & LI & Genus \\
\hline$(3 A, 3 A, 5 A)$ & -1 & 0 \\
$(3 A, 5 A, 5 A)$ & -1 & 1 \\
$(3 A, 5 A, 5 B)$ & 1 & 1 \\
$(5 A, 5 A, 5 A)$ & -1 & 2 \\
$(3 A, 3 A, 3 A, 3 A)$ & 1 & 0 \\
$(3 A, 5 A, 5 A, 5 A)$ & 1 & 3 \\
$(5 A, 5 A, 5 A, 5 A)$ & 1 & 4 \\
$(5 A, 5 A, 5 A, 5 B)$ & -1 & 4 \\
$(5 A, 5 A, 5 A, 5 A, 5 A)$ & -1 & 6
\end{tabular}

other cases, $\mathcal{H}^{\mathrm{in}}\left(A_{5}, \boldsymbol{C}\right)$ has exactly two components, which are distinguished by the lift invariant.

A theorem of Conway and Parker, whose detailed proof was supplied by Fried and Völklein in [8, says that, should the Schur multiplier of $G$ be generated by commutators, there exists some $K$ such that if $n_{C}>K$ for all conjugacy classes $C$ of $G$, then the inner Hurwitz space is connected. The Corollary can be seen as an effective version of the Conway-Parker-Fried-Völklein Theorem for the case of $G=A_{5}$. The combination of our result and the 3-cycle result of Fried suggests that the lift invariant may be a fine enough invariant to distinguish components of $\mathcal{H}^{\text {in }}\left(A_{n}, \boldsymbol{C}\right)$ for general $n$ and $\boldsymbol{C}$. The cases for small $n$ seem likely to prove crucial in any attempt at understanding the general structure of Hurwitz spaces. The main obstacle towards a classification for arbitrary $n$ is the need for greater insight into the structure of those types which do not fit the general rule, such as those listed in Lists A and B. The authors are currently working towards classification results for $A_{6}$ and $A_{8}$. Our most recent experiments suggest that the braids orbits for these groups are similarly organized.

Section 2 contains notation and preliminary results concerning braid groups and their interaction with partitions. We prove necessary preliminary results in Section 3. After that, for types with $3 \leq r \leq 7$, we quote an exhaustive search of all orbits with our MAPCLASS package for GAP 9,11. In particular, the search produced our Lists A and B of exceptional types. The proof of our Main Theorem can also be found in Section 3 . 


\section{BRAIDS AND PARTITIONS}

The proof of the Main Theorem requires us to consider subgroups of the braid group which fix a partition in a particular way. This section discusses such subgroups. The material in this section is a special case of the theory of mixed braids, in which, in the language of [10, all interior braids are trivial.

Definition 2.1. A partition of $\{1, \ldots, r\}$ will be called admissible if each block consists of a consecutive subsequence of the integers $1, \ldots, r$. Thus an admissible partition will have the form

$$
\left\{\left\{1, \ldots, n_{1}\right\},\left\{n_{1}+1, \ldots, n_{2}\right\}, \ldots,\left\{n_{k-1}+1, \ldots, r\right\}\right\},
$$

for some $1 \leq n_{1}<n_{2}<\cdots<n_{k-1}<r$. The notation $\left[a_{1}, a_{2}, \ldots, a_{k}\right]$ will be used to denote an admissible partition of $\{1, \ldots, r\}$ as above, where block $i$ has size $a_{i}$. In particular, the above partition can be written as

$$
\left[n_{1}, n_{2}-n_{1}, \ldots, r-n_{k-1}\right] .
$$

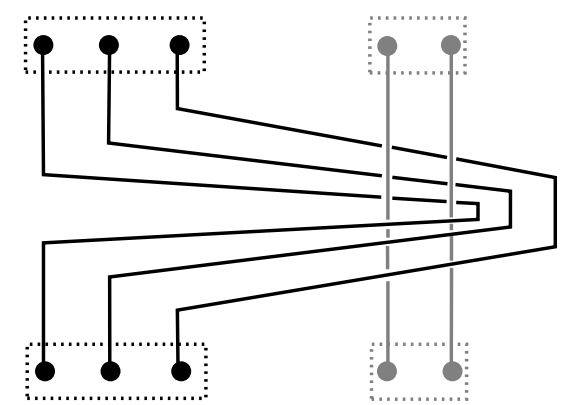

(a) The ribbon generator $R_{i, j}$. The elements of block $i$ are braided under block $j$ and all intermediate blocks.

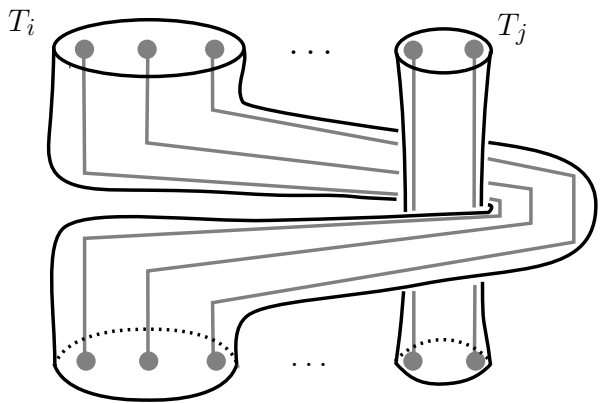

(b) Ribbon braids can be seen as braidings of blocks of braids. Strands in the same block lie in embedded cylinders.

FiguRE 1. Ribbon braids.

Fix an admissible partition $P$ of $\{1 \ldots, r\}$, with $k$ blocks $T_{1}, \ldots, T_{k}$. We say that a pure braid $Q \in \mathcal{P} \mathcal{B}_{r}$ is tubular with respect to $P$ if for each $1 \leq i \leq k$ we can embed a cylinder $D_{i} \cong D \times[0,1]$ into $\mathbb{C} \times[0,1]$ such that any two cylinders are disjoint and every strand corresponding to a point in $T_{i}$ lies in the interior of $D_{i}$ (see Figure 1b, a more thorough description can be found in 10]). To each cylinder $D_{i}$ we can associate a braid in $\mathcal{B}_{\left|T_{i}\right|}$ by restricting to the interior of $D_{i}$. Such a braid is called an interior braid. If a tubular braid with respect to $P$ is such that all interior braids are trivial, then we call this braid a ribbon braid. Define $\mathcal{P} \mathcal{R}_{r, P}$ to be the subgroup of $\mathcal{P} \mathcal{B}_{r}$ consisting of all ribbon braids, for the partition $P$. We call $\mathcal{P} \mathcal{R}_{r, P}$ the ribbon braid group with respect to $P$.

\section{Remark 2.2.}

- Ribbon braids preserve the partition $P$. In fact, they do more than that. The order of the strands within a block is preserved, since the braids are pure, and the order is preserved throughout time. The name ribbon braid 
is sometimes used to include the possibility that we rotate the strands in a cylinder by some multiple of $2 \pi([\underline{6})$. Our definition excludes such braids.

- We think of ribbon braids as those braids obtained by combining all the strands in a partition block into a single strand. For $1 \leq i<j \leq r$, define $R_{i, j}^{P}$ to be the ribbon braid which braids block $i$ under block $j$, in analogy with the pure braid generator $P_{i, j}$ (see Figure 1a).

The ribbon braid group $\mathcal{P} \mathcal{R}_{r, P}$ depends only on the number of blocks in the associated partition. The next proposition makes this relationship clear.

Proposition 2.3. Suppose that $P$ is an admissible partition of $\{1, \ldots, r\}$, and that $P$ has $k$ blocks. Then there is an isomorphism $\phi_{P}: \mathcal{P} \mathcal{R}_{r, P} \rightarrow \mathcal{P} \mathcal{B}_{k}$.

Proof. For each of the $k$ blocks select one representative strand, and discard all others. What remains is a pure braid on $k$ strands. This forgetful function $\phi_{P}$ : $\mathcal{P} \mathcal{R}_{r, P} \rightarrow \mathcal{P} \mathcal{B}_{r}$ is an isomorphism.

For the remainder of this section we want to examine ribbon braids and their action on tuples of length $r$.

Definition 2.4. Suppose $P=\left[n_{1}, \ldots, n_{k}\right]$ is an admissible partition of $\{1, \ldots, r\}$. Given a tuple $\boldsymbol{g}=\left(g_{1}, \ldots, g_{r}\right)$ of elements from a finite group $G$ we denote by $\boldsymbol{g}^{P}$ the tuple:

$$
\boldsymbol{g}^{P}=\left(g_{1} \cdots g_{n_{1}}, g_{n_{1}+1} \cdots g_{n_{1}+n_{2}}, \ldots, g_{n_{1}+\cdots+n_{k-1}+1} \cdots g_{r}\right) .
$$

We call such a tuple a coalesced tuple.

Coalescing reduces the size of the tuple whilst maintaining the product-one condition. However, if $\boldsymbol{g}$ is a Nielsen tuple, then $\boldsymbol{g}^{P}$ can fail to be a Nielsen tuple. One needs to ensure separately that none of the elements in $\boldsymbol{g}^{P}$ is the identity and that this tuple is generating.

The following observation does not require proof.

Lemma 2.5. Suppose that $P$ is an admissible partition of $\{1 \ldots, r\}$, with $k$ blocks. Let $R \in \mathcal{P R}_{r, P}, Q=\phi_{P}(R) \in \mathcal{P} \mathcal{B}_{k}$, and $\boldsymbol{g}=\left(g_{1}, \ldots, g_{r}\right)$ be a tuple of length $r$. Then

$$
(R(\boldsymbol{g}))^{P}=Q\left(\boldsymbol{g}^{P}\right) .
$$

It is clear that ribbon braids, as pure braids, act on the elements of the tuple by conjugation. Furthermore, the action on elements of the same partition block is identical.

Lemma 2.6. Let $P=\left[n_{1}, \ldots, n_{k}\right]$ be an admissible partition of $\{1, \ldots, r\}$. If $\boldsymbol{g}$ is a tuple of length $r$ and $R \in P R_{r, P}$, then

$$
R(\boldsymbol{g})=\left(g_{1}^{s_{1}}, \ldots, g_{m_{1}}^{s_{1}}, g_{m_{1}+1}^{s_{2}}, \ldots, g_{m_{2}}^{s_{2}}, \ldots, g_{m_{k-1}+1}^{s_{k}}, \ldots, g_{r}^{s_{k}}\right)
$$

for some $s_{i} \in G$. Here $m_{i}=n_{1}+\cdots+n_{i}$.

Proof. It suffices to consider the case where $R=R_{i, j}^{P}$ is one of the generators of $\mathcal{P} \mathcal{R}_{r, P}$, and then the claim is clear.

Under certain circumstances we may undo the coalescing in a unique way as the following proposition suggests. 
Proposition 2.7. Let $G$ be a finite group, $m$ a natural number, and $g \in G$ such that $(m, o(g))=1$. Suppose that $P=[\underbrace{1, \ldots, 1}_{i}, m, 1, \ldots, 1]$ is an admissible partition of $\{1, \ldots, r\}$ into $k=r-m+1$ blocks and that

$$
\begin{aligned}
\boldsymbol{g} & =(g_{1}, \ldots, g_{i}, \underbrace{g, \ldots, g}_{m}, g_{i+m+1}, \ldots, g_{r}) \\
\boldsymbol{g}^{\prime} & =(g_{1}^{\prime}, \ldots, g_{i}^{\prime}, \underbrace{g^{\prime}, \ldots, g^{\prime}}_{m}, g_{i+m+1}^{\prime}, \ldots, g_{r}^{\prime})
\end{aligned}
$$

are two tuples of the same type with $Q\left(\boldsymbol{g}^{P}\right)=\boldsymbol{g}^{\prime P}$ for some $Q \in \mathcal{P} \mathcal{B}_{k}$. Then $R(\boldsymbol{g})=\boldsymbol{g}^{\prime}$, where $R=\phi_{P}^{-1}(Q) \in \mathcal{P} \mathcal{R}_{r, P}$.

Proof. By Lemma 2.6, $R(\boldsymbol{g})=\left(h_{1}, \ldots, h_{i}, h, \ldots, h, h_{i+m+1}, \ldots, h_{r}\right)$ for suitable elements $h_{i}, h \in G$. Since $R(\boldsymbol{g})^{P}=Q\left(\boldsymbol{g}^{P}\right)=\boldsymbol{g}^{\boldsymbol{\prime}^{P}}$ by Lemma 2.5, it follows that $h_{j}=g_{j}^{\prime}$ for $1 \leq j \leq i$ and $i+m+1 \leq j \leq r$, and hence also that $h^{m}=\left(g^{\prime}\right)^{m}$. Since $o\left(g^{\prime}\right)=o(g)=o(h)$ and $(m, o(g))=1$, we have that $h=\boldsymbol{g}^{\prime}$ and so $R(\boldsymbol{g})=\boldsymbol{g}^{\prime}$.

\section{3. $A_{5}$ BRAID ORBITS}

In this section we aim to provide a complete description of braid orbits for $A_{5}$. For the rest of this article we will call an $r$-tuple of type $\boldsymbol{C}$ for which $n_{2 A}(\boldsymbol{C})>0$ an even tuple. Tuples of type $\boldsymbol{C}$ where $n_{2 A}(\boldsymbol{C})=0$ will be called odd tuples. By parity of a tuple we refer to whether the tuple is even or odd. The same terminology also applies to types.

First of all, in our proof we will consider the action of $\mathcal{P} \mathcal{B}_{r}$ instead of $\mathcal{B}_{r}$. Since $\mathcal{P} \mathcal{B}_{r}$ does not change the order of classes in $\boldsymbol{C}$, we need to introduce the "pure" version of the Nielsen classes.

For a type $\boldsymbol{C}$, we let the pure Nielsen class corresponding to $\boldsymbol{C}$ be

$$
\operatorname{PNi}^{\text {in }}(G, \boldsymbol{C})=\left\{\boldsymbol{g} \in N(G) \mid g_{i} \in C_{i} \text { for all } i\right\} .
$$

Clearly, $\mathcal{P} \mathcal{B}_{r}$ acts on $\mathrm{PNi}^{\mathrm{in}}(G, C)$. The orbits under this action (as well as the action of $\left.\operatorname{Inn}(G) \times \mathcal{P} \mathcal{B}_{r}\right)$ will be called the pure braid orbits.

Every braid $Q \in \mathcal{B}_{r}$ acting on a tuple of type $\boldsymbol{C}$ produces a tuple of type $\boldsymbol{C}^{\sigma_{Q}}$, where $\sigma_{Q} \in S_{r}$ is the permutation associated with $Q$. Consequently, we have:

Lemma 3.1. The action of $Q \in \mathcal{B}_{r}$ on $\mathrm{PNi}^{\mathrm{in}}(G, C)$ is a bijection between this class and the class $\mathrm{PNi}^{\mathrm{in}}\left(G, C^{\sigma_{Q}}\right)$. This bijection takes pure braid orbits to pure braid orbits and, for odd types, it preserves the lift invariant.

This lemma will help us deduce our Main Theorem from the following "pure braid" equivalent.

Theorem 3.2. For $G=A_{5}$ and a type $\boldsymbol{C}=\left(C_{1}, \ldots, C_{r}\right), r \geq 3$, the pure Nielsen class $\mathrm{PNi}^{\mathrm{in}}(G, \boldsymbol{C})$ is non-empty if and only if $\boldsymbol{C}$ is not on List $A$. Furthermore, if non-empty, $\mathrm{PNi}^{\mathrm{in}}(G, C)$ contains at most two pure braid orbits, and

- for even types $\boldsymbol{C}, \mathcal{P} \mathcal{B}_{r}$ acts transitively on $\mathrm{PNi}^{\mathrm{in}}(G, C)$;

- for odd types $\boldsymbol{C}$, there are two pure braid orbits on $\mathrm{PNi}^{\mathrm{in}}(G, C)$ if and only if $\boldsymbol{C}$, up to permutation, is not listed in List B; moreover, the two orbits are distinguished by the lift invariant. 
We now commence proving Theorem 3.2

3.1. Basis for induction. The proof is by induction on $r$, the length of $\boldsymbol{C}$. The following lemma anchors the induction.

Lemma 3.3. If $\boldsymbol{C}$ is a type of length $3 \leq r \leq 7$, then the conclusion of Theorem 3.2 holds.

The lemma is established by explicit calculation of all orbits for all types of length $3 \leq r \leq 7$. Note that in view of Lemma 3.1, we only need to consider lexicographically ordered types. For these types, the computation was done using our MAPClAss package ([11]) for the GAP computational algebra system ([9]). Data for this computation is available upon request. More details about how such a computation is performed can be found in [14. In particular, all exceptional cases were found in this computation.

In the introduction we commented that Fried's 3-cycle result gives a succinct rule for when the braid group acts transitively: if the genus of the corresponding cover is 0 . An inspection of the genera of the exceptional types in List A demonstrates that this rule does not extend to all types. Furthermore, notice that it would be possible to predict some of the types for which $\mathrm{Ni}^{\text {in }}(G, C)$ is empty, since this must be the case if the genus is negative. Finally we remark that if the genus of the cover is zero, then there exists an easy to calculate formula for the lift invariant [7]. Our calculations agree with this formula in those cases.

\subsection{A normal form for tuples.}

Definition 3.4. We say that a tuple

$$
\left(g_{1}, \ldots, g_{i-1}, g, g, g_{i+2}, \ldots, g_{r}\right)
$$

is in odd repetitive form if $g$ is an odd-order element. If the position of the repeated element is important, then we shall say that the tuple is in odd repetitive form at position $i$. A tuple

$$
\left(g_{1}, \ldots, g_{i-1}, g, g, g, g_{i+3}, \ldots, g_{r}\right)
$$

where $g$ is a double transposition, is said to be in even repetitive form at position $i$.

For a tuple $\boldsymbol{g}$ in repetitive form at position $i$, define $P_{\boldsymbol{g}}$ to be the partition $[\underbrace{1, \ldots, 1}_{i-1}, 2,1, \ldots, 1]$ or $[\underbrace{1, \ldots, 1}_{i-1}, 3,1, \ldots, 1]$, depending on the parity of the form.

Similarly, we will talk about types in odd or even repetitive form at position $i$. In the first case, this means that $C_{i}=C_{i+1}$ is an odd-order class; in the second, $C_{i}=C_{i+1}=C_{i+2}$ is the double-transposition class.

Nielsen tuples in repetitive form are useful for our induction because coalescing such tuples with respect to $P=P_{\boldsymbol{g}}$ preserves all the salient properties. The following lemma makes this precise. Note, first of all, that the coalesced element $g^{2}$ or $g^{3}$ in the respective cases is not identity.

Recall that, for every odd order element $g \in A_{5}$, the unique odd order lift of $g$ to $2 \cdot A_{5}$ is denoted by $\hat{g}$.

Lemma 3.5. Suppose $\boldsymbol{g}$ is a tuple in a repetitive form, $P=P_{\boldsymbol{g}}$, and $\boldsymbol{h}=\boldsymbol{g}^{P}$. Then $\boldsymbol{g}$ is a Nielsen tuple if and only if $\boldsymbol{h}$ is a Nielsen tuple. Furthermore, $\boldsymbol{g}$ and $\boldsymbol{h}$ have the same parity. If they are odd, then $L I(\boldsymbol{g})=L I(\boldsymbol{h})$. 
Proof. The second statement is obvious. For the first claim, if $\boldsymbol{g}$ is in odd repetitive form at position $i$, then, since $g_{i}=g_{i+1}$ is of odd order, $\left\langle g_{i}, g_{i+1}\right\rangle=\left\langle g_{i} g_{i+1}\right\rangle$, and this yields the claim. If $\boldsymbol{g}$ is in even repetitive form, then $\boldsymbol{g}$ and $\boldsymbol{h}$ contain the same elements and so again the claim follows.

Now suppose $\boldsymbol{g}$ is odd and, in particular, it is in odd repetitive form at position $i$. Let $g=g_{i}=g_{i+1}$. Note that $\widehat{g^{2}}=(\hat{g})^{2}$. Therefore,

$$
\begin{aligned}
L I(\boldsymbol{g}) & =\hat{g}_{1} \cdots \hat{g}_{i-1}(\hat{g} \hat{g}) \hat{g}_{i+2} \cdots \hat{g}_{r} \\
& =\hat{g}_{1} \cdots \hat{g}_{i-1} \widehat{\left(g^{2}\right)} \hat{g}_{i+2} \cdots \hat{g}_{r} \\
& =L I(\boldsymbol{h}),
\end{aligned}
$$

completing the proof.

In the remainder of this subsection we prove the following.

Proposition 3.6. Let $\boldsymbol{C}=\left(C_{1}, \ldots, C_{r}\right), r \geq 6$, be a type in a repetitive form at position $i$. Then

- either every pure braid orbit on $\mathrm{PNi}^{\mathrm{in}}(G, C)$ contains a tuple in repetitive form at position $i$,

- or $r=6$ and either

- $\boldsymbol{C}=(2 A, 2 A, 2 A, 2 A, 2 A, 2 A)$, or

- $\boldsymbol{C}=(5 A, 5 A, 5 A, 5 A, 5 B, 5 B)$ or $(5 A, 5 A, 5 B, 5 B, 5 B, 5 B)$ up to permutation and, furthermore, $C_{i}=C_{i+1}=5 B$ or $5 A$, respectively.

The proof of this proposition will be given in three lemmas. Throughout the proof we assume the hypotheses of Proposition 3.6 and each lemma establishes the conclusion of the proposition for different values of $r$ and $i$.

Lemma 3.7. The claim holds if $r \leq 7$.

Proof. Consider a pure braid orbit $\Omega$ on $\operatorname{PNi}^{\text {in }}(G, C)$.

Suppose first that $C=C_{i}=C_{i+1}$ is an odd order class. Define the type

$$
\boldsymbol{D}=\left(C_{1}, \ldots, C_{i-1}, C^{2}, C_{i+2}, \ldots, C_{r}\right),
$$

where $C^{2}$ is the class containing the squares of elements from $C$. Hence, if $C=3 A$, then also $C^{2}=3 A$. If $C=5 A$ (respectively, $5 B$ ), then $C^{2}=5 B$ (respectively, $5 A$ ).

Note that the types $\boldsymbol{C}$ and $\boldsymbol{D}$ have the same parity. If they are even, then there is only one orbit on $\operatorname{PNi}^{\text {in }}(G, C)$ by Lemma 3.3. Thus, $\Omega=\operatorname{PNi}^{\text {in }}(G, C)$. Since $\boldsymbol{D}$ has length $r-1 \geq 5$, the pure Nielsen class $\mathrm{PNi}^{\mathrm{in}}(G, \boldsymbol{D})$ is not empty. Select any tuple $\boldsymbol{g}=\left(g_{1}, \ldots, g_{i-1}, g, g_{i+2}, \ldots, g_{r}\right)$ from this class. Since $g$ has odd order, there is a unique $h \in\langle g\rangle$ such that $g=h^{2}$. Note that this $h$ is contained in $C$ and so the tuple $\boldsymbol{h}=\left(g_{1}, \ldots, g_{i-1}, h, h, g_{i+2}, \ldots, g_{r}\right)$ is of type $\boldsymbol{C}$. By Lemma 3.5 since $\boldsymbol{g}$ is a Nielsen tuple, $\boldsymbol{h}$ must also be a Nielsen tuple. Hence $\boldsymbol{h}$ is in $\Omega$ and it is evidently in odd repetitive form at position $i$.

Now suppose that $\boldsymbol{C}$ and $\boldsymbol{D}$ are odd. By Lemma 3.3, $\Omega$ is one of two pure braid orbits on $\mathrm{PNi}^{\text {in }}(G, C)$, and let $\epsilon$ be the lift invariant of $\Omega$. Note that $\boldsymbol{D}$ is on List $\mathrm{B}$ only if it is $(5 A, 5 A, 5 A, 5 A, 5 A)$ or $(5 B, 5 B, 5 B, 5 B, 5 B)$. This leads to the second exceptional case above. Otherwise, the same Lemma 3.3 implies that there is a pure braid orbit on $\operatorname{PNi}^{\text {in }}(G, \boldsymbol{D})$ with lift invariant $\epsilon$. Let $\boldsymbol{g}=$ $\left(g_{1}, \ldots, g_{i-1}, g, g_{i+2}, \ldots, g_{r}\right)$ be a tuple from that orbit. As above, select $h \in\langle g\rangle$ such that $g=h^{2}$ and set $\boldsymbol{h}=\left(g_{1}, \ldots, g_{i-1}, h, h, g_{i+2}, \ldots, g_{r}\right)$. Again, we note that 
$\boldsymbol{h}$ is a Nielsen tuple of type $\boldsymbol{C}$. Furthermore, by Lemma 3.5, it has lift invariant $\epsilon$. Therefore, $\boldsymbol{h}$ is in $\Omega$ and it is in the required repetitive form.

Finally, suppose $C$ is the double-transposition class. Let

$$
\boldsymbol{D}=\left(C_{1}, \ldots, C_{i-1}, C, C_{i+3}, \ldots, C_{r}\right) .
$$

Then $\boldsymbol{D}$ is an even type of length at least four. If it is on List A, it must be $(2 A, 2 A, 2 A, 2 A)$, leading to the first exceptional case above. Otherwise, $\mathrm{PNi}^{\mathrm{in}}(G, \boldsymbol{D})$ is non-empty. Select $\boldsymbol{g}=\left(g_{1}, \ldots, g_{i-1}, g, g_{i+3}, \ldots, g_{r}\right) \in \mathrm{PNi}^{\text {in }}(G, \boldsymbol{D})$ and set $\boldsymbol{h}=\left(g_{1}, \ldots, g_{i-1}, g, g, g, g_{i+3}, \ldots, g_{r}\right)$. Clearly, $\boldsymbol{h}$ is a Nielsen tuple of type $\boldsymbol{C}$. Since $\boldsymbol{C}$ is even, $\Omega=\operatorname{PNi}^{\text {in }}(G, C)$ by Lemma 3.3, and so $\boldsymbol{g}$ is in $\Omega$.

We note that all exceptions in this lemma are bona fide, that is, for each of these types there exists a pure braid orbit containing no tuple in repetitive form at position $i$.

Next, let us consider the case where the repeated classes are at the end of the type.

Lemma 3.8. Let $r \geq 8$ and suppose that $i=r-1$ in the odd repetitive form case and $i=r-2$ in the even repetitive form case. Then the claim holds.

Proof. We are proving this by induction on $r$, with $r=6$ and 7 serving as base cases. Let $\Omega$ be a pure braid orbit of type $\boldsymbol{C}$. Select a tuple $\boldsymbol{g}=\left(g_{1}, \ldots, g_{r}\right) \in \Omega$. We first claim that $\boldsymbol{g}$ can be chosen so that $g_{r-1}$ and $g_{r}$ do not commute. Indeed, if this is not the case, then $g_{r-1} \in C=C_{G}\left(g_{r}\right)$. Since $\boldsymbol{g}$ is generating, there is $g_{j}$ that does not normalize $C$. Applying the pure braid $P_{j, r-1}$, we obtain a new tuple from $\Omega$, where in position $r-1$ we find $g_{r-1}^{g_{j}^{-1}}$, which is not in $C$. Here we use the property of $G=A_{5}$ that $C^{g}$ is either equal to $C$ or intersects $C$ trivially.

From now on we assume that $g_{r-1}$ and $g_{r}$ do not commute. In particular, $\left\langle g_{r-1}, g_{r}\right\rangle$ is either a maximal subgroup of $G$ or it is all of $G$. (This is again a property of $G=A_{5}$.) We will call the repeated positions, $[i, \ldots, r]$, at the end the special positions and all others general.

We will first try to coalesce $\boldsymbol{g}$ using two adjacent general positions, $j$ and $j+1$. Hence $j<i-1$ and the partition used is $P=[\underbrace{1, \ldots, 1}_{j-1}, 2,1, \ldots, 1]$. Suppose that the resulting tuple $\boldsymbol{h}=\left[g_{1}, \ldots, g_{j-1}, g_{j} g_{j+1}, g_{j+2}, \ldots, g_{r}\right)$ is a Nielsen tuple. Since $r-1 \geq 7$, by induction, there exists a pure braid $Q \in \mathcal{P} \mathcal{B}_{r-1}$ such that $Q(\boldsymbol{h})$ is in repetitive form at position $i-1$. Let $R \in \mathcal{P} \mathcal{R}_{r, P}$ be the ribbon braid such that $\phi_{P}(R)=Q$. By Lemma 2.5, $(R(\boldsymbol{g}))^{P}=Q(\boldsymbol{h})$. This means that $R(\boldsymbol{g})$ is in repetitive form at position $i$, and so the claim holds.

We will now see that if this trick does not work for $\boldsymbol{g}$ for any $j$, then this tuple has a very restricted shape.

In view of our choice, since $\boldsymbol{g}$ is generating, there is a general position $k$ such that $g_{k}$ and the elements in the special positions generate $G$. By the above, we can assume now that $g_{j} g_{j+1}=1$ for any two adjacent general positions disjoint from $k$. Indeed, if $g_{j} g_{j+1} \neq 1$, then the coalesced tuple $\boldsymbol{h}$ is a Nielsen tuple, as it clearly contains generating elements for $G$. Hence, $g_{j+1}=g_{j}^{-1}$ for all $j$ as above. Since $r \geq 8$, there are at least five general positions. This means that either in front of $g_{k}$ or after $g_{k}$ there are at least two general positions. The proof is symmetric for these two possibilities, so let us assume the former. Hence $k \geq 3$. 
We know that $g_{j+1}=g_{j}^{-1}$ for all $j<k-1$. We claim that the same holds for $j=$ $k-1$. If not, the tuple $\boldsymbol{h}$ does not contain the identity element and it is generating, since $\left\langle g_{k-2}, g_{k-1}, g_{k}\right\rangle=\left\langle x, x^{-1}, g_{k}\right\rangle=\left\langle x, x^{-1} g_{k}\right\rangle=\left\langle g_{k-2}, g_{k-1} g_{k}\right\rangle$. Thus, $g_{k}=$ $g_{k-1}^{-1}$. Therefore, in $\boldsymbol{g}$ we have the sequence $x, x^{-1}, x, x^{-1}, \ldots$ in positions 1 through $k$. In particular, $g_{1}=g_{k}$ or $g_{k}^{-1}$. This means that we could have chosen $k=1$. Now applying the symmetric argument (for the general positions after $g_{1}$ ), we conclude the all elements in the general positions obey the same pattern $x, x^{-1}, x, x^{-1}, \ldots$

Now we try another trick, namely, we coalesce with respect to $P=[3,1, \ldots, 1]$. This gives $\boldsymbol{h}=\left(x, g_{4}, \ldots, g_{r}\right)$, which is clearly a Nielsen tuple. Note that this time $\boldsymbol{h}$ has length $r-2 \geq 6$. The above inductive argument with the pure braid $Q$ and the corresponding ribbon braid $R$ works whenever $\boldsymbol{h}$ does not fall into one of the exceptional cases from Proposition 3.6. In all these cases $r-2=6$ and so $r=8$.

We deal with the two exceptional cases in turn. Suppose that $\boldsymbol{h}$ is of type $(2 A, 2 A, 2 A, 2 A, 2 A, 2 A)$ and so $\boldsymbol{g}$ is of type $(2 A, 2 A, 2 A, 2 A, 2 A, 2 A, 2 A, 2 A)$. By the above, $\boldsymbol{g}=\left(x, x, x, x, x, g_{6}, g_{7}, g_{8}\right)$. This implies that the tuple $\left(x, g_{6}, g_{7}, g_{8}\right)$ is a Nielsen tuple of type $(2 A, 2 A, 2 A, 2 A)$. However, this is impossible, since this type is on List A and so the corresponding pure Nielsen class is empty.

In the second exceptional case $\boldsymbol{h}$ is odd and so $\boldsymbol{g}$ is odd, too. By the above, $\boldsymbol{g}=\left(x, x^{-1}, x, x^{-1}, x, x^{-1}, g_{7}, g_{8}\right)$. Now the product-one condition implies that $g_{7} g_{8}=1$, which is a contradiction, since $g_{7}$ and $g_{8}$ do not commute. This completes the proof.

Finally, we can do the general case.

Lemma 3.9. If $r \geq 8$, then the claim holds.

Proof. Consider $Q=Q_{1} \cdots Q_{r-1} \in \mathcal{B}_{r}$. This braid rotates each tuple, sending $\left(g_{1}, \ldots, g_{r}\right)$ to $\left(g_{r}, g_{1}, \ldots, g_{r-1}\right)$.

Now suppose that $\Omega$ is a pure braid orbit of type $\boldsymbol{C}$ and let $\boldsymbol{g}=\left(g_{1}, \ldots, g_{r}\right) \in \Omega$. Let $j$ be the final position of our repeated classes in $\boldsymbol{C}$. So $j=i+1$ in the odd repetitive form case and $j=i+2$ in the even repetitive form case. Note that $Q^{r-j}(\boldsymbol{g})$ is of type $\boldsymbol{C}^{\sigma^{r-j}}$, where $\sigma=\sigma_{Q}$ is the cycle $(1,2, \ldots, r) \in S_{r}$. Hence the repeated classes are now at the end of the tuple and so Lemma 3.8 applies. Therefore, there exists a pure braid $R$ such that $R Q^{r-j}(\boldsymbol{g})$ has the repeated elements in the last two or three positions depending on the form parity. Finally, $Q^{-(r-j)} R Q^{r-j}(\boldsymbol{g})$ is again of type $C$ and it is in the required repetitive form at position $i$.

It remains to notice that $Q^{-(r-j)} R Q^{r-j}=R^{Q^{r-j}}$ is a conjugate of $R$ and hence it is a pure braid.

This completes the proof of Proposition 3.6 .

3.3. Proof of Theorem 3.2 and the Main Theorem. Let $r \geq 8$. In view of Lemma 3.1, we can assume that $\boldsymbol{C}=\left(C_{1}, \ldots, C_{r}\right)$ is lexicographically ordered.

We begin by considering the question of existence.

Lemma 3.10. There exists a Nielsen tuple of type $\boldsymbol{C}$. Moreover, if the type is odd, then there are Nielsen tuples for both possible values of the lift invariant.

Proof. We use induction on $r$. Since $r \geq 8, C$ must contain some conjugacy class twice. Since $\boldsymbol{C}$ is lexicographically ordered, we can assume that $C_{i}=C_{i+1}$. The type $\boldsymbol{D}=\left(C_{1}, \ldots, C_{i-1}, C_{i+2}, \ldots, C_{r}\right)$ is of length at least $r-2 \geq 6$ and lexicographically ordered. Hence by induction (and since List A contains no type of such length) 
there exists a Nielsen tuple $\left(g_{1}, \ldots, g_{i-1}, g_{i+2}, \ldots, g_{r}\right)$ of type $\boldsymbol{D}$. Pick $g \in C_{i}$ and note that $g^{-1} \in C_{i}=C_{i+1}$. Therefore the tuple $\left(g_{1}, \ldots, g_{i-1}, g, g^{-1}, g_{i+2}, \ldots, g_{r}\right)$ is a Nielsen tuple of type $\boldsymbol{C}$.

Suppose now that $C$ is odd and let $\epsilon \in\{1,-1\}$. We proceed in exactly the same way as above. By induction (and since List B contains no type of length $r-2 \geq 6)$ we can select $\left(g_{1}, \ldots, g_{i-1}, g_{i+2}, \ldots, g_{r}\right)$ with lift invariant $\epsilon$, in which case the extended tuple $\left(g_{1}, \ldots, g_{i-1}, g, g^{-1}, g_{i+2}, \ldots, g_{r}\right)$ also has lift invariant $\epsilon$, since $\widehat{g^{-1}}=(\hat{g})^{-1}$.

We can now complete the proof of Theorem 3.2

Proof of Theorem 3.2, Let $\boldsymbol{g}=\left(g_{1}, \ldots, g_{r}\right)$ and $\boldsymbol{g}^{\prime}=\left(g_{1}^{\prime}, \ldots, g_{r}^{\prime}\right)$ be two Nielsen tuples of type $\boldsymbol{C}$. If the type is odd let us assume that they have the same lift invariant. We need to show that there exists a pure braid $R \in \mathcal{P} \mathcal{B}_{r}$ such that $R(\boldsymbol{g})=\boldsymbol{g}^{\prime}$.

If $\boldsymbol{C}$ has the same odd class twice, then, since $\boldsymbol{C}$ is lexicographically ordered, it is in odd repetitive form at some position $i$. Otherwise, $C$ contains no more than three odd classes and hence it contains the double-transposition class at least five times. In particular, $\boldsymbol{C}$ in this case is in even repetitive form at position $i=1$. In either case, by Proposition [3.6. each of $\boldsymbol{g}$ and $\boldsymbol{g}^{\prime}$ are conjugate by pure braids to some Nielsen tuples in repetitive form at position $i$. Hence, without loss of generality we can assume that $\boldsymbol{g}$ and $\boldsymbol{g}^{\prime}$ are themselves in this form.

Let $P=P_{\boldsymbol{g}}=[\underbrace{1, \ldots, 1}_{i-1}, 2,1, \ldots, 1]$ or $[3,1, \ldots, 1]$ depending on the case. Let $\boldsymbol{h}=\boldsymbol{g}^{P}$ and $\boldsymbol{h}^{\prime}=\left(\boldsymbol{g}^{\prime}\right)^{P}$. By Lemma 3.5, $\boldsymbol{h}$ and $\boldsymbol{h}^{\prime}$ are Nielsen tuples and if they are odd, then they have the same lift invariant. Clearly, $\boldsymbol{h}$ and $\boldsymbol{h}^{\prime}$ are of the same type. Note that in the odd form case the new type may not be lexicographically ordered! This does not matter since the statement of Theorem 3.2 does not require this. We also note that by a more careful choice of $i$ we could in fact ensure that the coalesced type be lexicographically ordered.

By induction and since $r-2 \geq 6, \boldsymbol{h}$ and $\boldsymbol{h}^{\prime}$ lie in the same pure braid orbit and hence there exists a pure braid $Q \in \mathcal{P} \mathcal{B}_{r-1}$ or $\mathcal{P} \mathcal{B}_{r-2}$, respectively, such that $Q(\boldsymbol{h})=\boldsymbol{h}^{\prime}$. Let $R \in \mathcal{P} \mathcal{R}_{r, P}$ such that $\phi_{P}(R)=Q$. By Proposition 2.7, $R(\boldsymbol{g})=\boldsymbol{g}^{\prime}$.

It remains to discuss how Theorem 3.2 implies the Main Theorem. For each type $\boldsymbol{C}$, the Nielsen class $\mathrm{Ni}^{\mathrm{in}}(G, \boldsymbol{C})$, when non-empty, is the union of the pure Nielsen classes $\mathrm{PNi}^{\text {in }}\left(G, C^{\sigma}\right)$ for all $\sigma \in S_{r}$. If $\boldsymbol{C}$ is even, then $\mathcal{P} \mathcal{B}_{r}$ acts transitively on each of these pure Nielsen classes, while $\mathcal{B}_{r}$ also fuses them together into a single braid orbit (see Lemma 3.1), since $\mathcal{B}_{r}$ maps to $S_{r}$ surjectively.

Similarly, if $\boldsymbol{C}$ is odd and $\epsilon \in\{1,-1\}$, then $\mathcal{B}_{r}$ fuses the pure braid orbits with lift invariant $\epsilon$ (when such orbits exist) into a single braid orbit with lift invariant $\epsilon$. Again, this follows from Lemma 3.1 .

This completes the proof of the Main Theorem.

\section{ACKNOWLEDGEMEnts}

The first author was supported by an EPSRC doctoral training grant. The second and third authors wish to thank Michael D. Fried and Helmut Völklein for generously sharing their ideas on Hurwitz spaces, the lift invariant, and the Conway-Parker Theorem. Without them the present paper would certainly not 
exist. Finally, the authors would like to thank the referee for the very helpful comments and suggestions.

\section{REFERENCES}

[1] Joan S. Birman, Braids, links, and mapping class groups, Princeton University Press, Princeton, N.J., 1974. Annals of Mathematics Studies, No. 82. MR0375281 (51 \#11477)

[2] Fabrizio Catanese, Michael Loenne, and Fabio Perroni, The irreducible components of the moduli space of dihedral covers of algebraic curves, preprint, arXiv:1206.5498 (2012).

[3] Fabrizio Catanese, Michael Lönne, and Fabio Perroni, Irreducibility of the space of dihedral covers of the projective line of a given numerical type, Atti Accad. Naz. Lincei Cl. Sci. Fis. Mat. Natur. Rend. Lincei (9) Mat. Appl. 22 (2011), no. 3, 291-309, DOI 10.4171/RLM/601. MR2847474(2012m:14057)

[4] A. Clebsch, Theorie der binaren algebraischen formen, 1872.

[5] J. H. Conway, R. T. Curtis, S. P. Norton, R. A. Parker, and R. A. Wilson, Atlas of finite groups, Maximal subgroups and ordinary characters for simple groups; With computational assistance from J. G. Thackray. Oxford University Press, Eynsham, 1985. MR.827219 (88g:20025)

[6] Roger Fenn, Dale Rolfsen, and Jun Zhu, Centralisers in the braid group and singular braid monoid, Enseign. Math. (2) 42 (1996), no. 1-2, 75-96. MR1395042 (97h:20047)

[7] Michael D. Fried, Alternating groups and moduli space lifting invariants, Israel J. Math. 179 (2010), 57-125, DOI 10.1007/s11856-010-0073-2. MR2735035 (2012a:14055)

[8] Michael D. Fried and Helmut Völklein, The inverse Galois problem and rational points on moduli spaces, Math. Ann. 290 (1991), no. 4, 771-800, DOI 10.1007/BF01459271. MR $1119950(93 \mathrm{a}: 12004)$

[9] The GAP Group, GAP - Groups, Algorithms, and Programming, Version 4.4.12, 2008.

[10] Juan González-Meneses and Bert Wiest, On the structure of the centralizer of a braid (English, with English and French summaries), Ann. Sci. École Norm. Sup. (4) 37 (2004), no. 5, 729-757, DOI 10.1016/j.ansens.2004.04.002. MR.2103472(2005h:20089)

[11] A. James, K. Magaard, S. Shpectorov, and H. Völklein, MAPCLASS: A package for mapping class orbit computation, 2011.

[12] Gareth A. Jones and Alexander Zvonkin, Orbits of braid groups on cacti (English, with English and Russian summaries), Mosc. Math. J. 2 (2002), no. 1, 127-160, 200. MR.1900588 (2003h:20067)

[13] Fu Liu and Brian Osserman, The irreducibility of certain pure-cycle Hurwitz spaces, Amer. J. Math. 130 (2008), no. 6, 1687-1708, DOI 10.1353/ajm.0.0031. MR2464030 (2009h:14052)

[14] Kay Magaard, Sergey Shpectorov, and Helmut Völklein, A GAP package for braid orbit computation and applications, Experiment. Math. 12 (2003), no. 4, 385-393. MR2043989 (2005e:12007)

[15] Charmaine Sia, Hurwitz equivalence in tuples of dihedral groups, dicyclic groups, and semidihedral groups, Electron. J. Combin. 16 (2009), no. 1, Research Paper 95, 17. MR2529804 (2010i:20041)

[16] Helmut Völklein, Moduli spaces for covers of the Riemann sphere, Israel J. Math. 85 (1994), no. 1-3, 407-430, DOI 10.1007/BF02758650. MR1264353 (95c:14022)

[17] Helmut Völklein, Groups as Galois groups, An introduction. Cambridge Studies in Advanced Mathematics, vol. 53, Cambridge University Press, Cambridge, 1996. MR 1405612 (98b:12003)

School of Mathematics, University of Birmingham, Birmingham, B15 2TT, United KINGDOM

E-mail address: adamjames87@gmail.com

School of Mathematics, University of Birmingham, Birmingham, B15 2TT, United KINGDOM

E-mail address: k.magaard@bham.ac.uk

School of Mathematics, University of Birmingham, Birmingham, B15 2TT, United KINGDOM

E-mail address: S.Shpectorov@bham.ac.uk 